\title{
Islami(sti)scher Rap in Deutschland? Sozial- und kulturwissenschaftliche Beobachtungen zum Diskurs um Integrationsverweigerung und Fundamentalismus
}

Marc Dietrich und Martin Seeliger

\section{Einleitung}

Wenn jemand fragt, was der Euro-Rettungsschirm, Marco Weiß im türkischen Jugendgefängnis ${ }^{1}$ und die Lebenssituation von Muslimen in Deutschland gemeinsam haben, könnte dies dem ersten Eindruck nach vielleicht etwas befremdlich erscheinen. Solche Verwirrungen würden sicherlich nicht zu Unrecht hervorgerufen, liegt doch die Verknüpfungsleistung nicht bei gegenstandsimmanenten Überschneidungen supranationaler Wirtschaftspolitik, juristischer Aufarbeitung sexueller Gewalt und gruppenspezifischer Lebensstile. Um die Verbindung der drei Sachverhalte erkennen zu können, muss man sich - so unsere These - zuerst einmal in einer Weise von ihnen distanzieren, die nicht sie selbst in den Mittelpunkt des Interesses rückt, sondern vielmehr die Art, mit der sie öffentlich diskutiert werden. Etwas bündiger und mit Niklas Luhmann formuliert: Es bedarf neben der Analyse der Oberfläche einer „Beobachtung zweiter Ordnung“. 2

Stellt man nun die Frage abgewandelt nach etwaigen Gemeinsamkeiten fauler sog. griechischer Arbeiter, inkompetenter türkischer Justiz und Muslimen in Deutschland, wird der umfassende Referenzrahmen deutlicher. Während die Eigenheit, dass alle drei Phänomene einen (wie auch immer gearteten) Bezug zur deutschen Gesellschaft aufweisen, noch relativ wenig Aussagekraft entfalten kann, findet sich eine Gemeinsamkeit in den Zuschreibungen, die die drei Phänomene in der öffentlichen Diskussion erfuhren und erfahren. Dass diese

\footnotetext{
${ }^{1}$ Vgl. Marco Weiß 2008.

${ }^{2}$ Niklas Luhmann 2008, S. 434.
} 
nicht eben positiv, sondern vor allem pathologisch ausfallen, nehmen wir im vorliegenden Text als relevante Rahmenbedingung unserer Analyse des Verhältnisses von Rap und Islam.

Konstatieren lässt sich zunächst und auch mit Verweis auf die Eingangsbemerkungen, dass „Islam und Deutschland“ ein prekäres Thema ist: Die Verteilung von Koran-Exemplaren und die immer wieder aufflammenden Diskussion um den Moscheebau in Köln, ${ }^{3}$ an der gesellschaftliche Akteure verschiedenster Couleur teilnahmen, sich Provokationen sowie gewaltsamen Ausschreitungen in eben diesen Fällen abspielten, kann man feststellen: Islam und Deutschland - dass ist auch und aktuell ein gesellschaftlich polarisierendes Thema. Nun wollen wir in diesem Beitrag weniger das vermeintlich generelle Verhältnis von einer „deutschen“ und einer ,islamischen Kultur" rekonstruieren, als vielmehr einen Blick in die Popkultur wagen. Hierbei handelt es sich keineswegs um ein Phänomen, das einer reinen „Oberflächenästhetik“ verschrieben ist, ${ }^{4}$ sondern durchaus um ein komplexes Netz aus Symbolen und Narrativen, das auf gesellschaftlich relevanten und fundierten Diskursen aufruht - mit der Folge, dass auch politische oder religiöse Semantiken im Pop ästhetisiert und verhandelt werden. Dies gilt besonders für Rap als einer ästhetischen Praxis, dem der Ruf einer subversiven Kultur, als Sprachrohr marginalisierter Gruppen, anhaftet(e). Die Erkenntnis einer politischen Dimension von Pop und Rap ist weder neu noch überraschend. Sowohl im Feuilleton als auch in der Wissenschaft wird dem Rechnung getragen - man denke an die wegweisenden Arbeiten der Cultural Studies. Pop im Allgemeinen und HipHop im Besonderen ist hier längst ein konstantes Thema der Auseinandersetzung. ${ }^{5}$

Eine politische Imprägnierung des vermeintlich hedonistischen Pop-Programms lässt sich also in ganz besonderer Weise für Rap ${ }^{6}$ konstatieren. ${ }^{7} \mathrm{Ne}-$

\footnotetext{
${ }^{3}$ Vgl. den Beitrag von Uwe Gerrens (S. 335-367) in diesem Sammelband.

${ }^{4}$ Thomas Hecken 2009, S. 265-271.

${ }^{5}$ Vgl. Rupa Huq 2007, S. 78.

${ }^{6}$ Wir sprechen von Rap als der musikalischen Praxis, die in der übergreifenden HipHop-Kultur integriert ist. Neben den klassischen vier Elementen (Breakdance, Graffiti, DJing/Turntablelism und eben Rap) lassen sich mittlerweile auch noch weitere ausdifferenzierte Disziplinen nennen. In diesem Artikel fokussieren wir allerdings auf Rapmusik.

${ }^{7}$ Detaillierte Ausführungen zu HipHop und Rap werden wir an dieser Stelle nicht liefern, histori-
} 
ben den Topoi der seit ein paar Jahren dominanten und mittlerweile wieder abgeklungenen „Bling-Bling-Ära“, die durchaus auch eine politische Komponente hatte, ${ }^{8}$ gibt und gab es immer auch politischen Rap, der jedoch selten jene Popularität erlangte wie die Protagonisten des erwähnten Zeitalters.

\section{Krisendiskurs:}

\section{Akteure (und Rapper) mit Migrationshintergrund}

Im Fokus der Medien stehen immer wieder Rapper, deren auf Authentizität getrimmte Texte, deren Spiel mit Fiktion und Biographie zu einer Verwirrung der Rezipienten führt. Wo fängt das künstlerische Alter Ego an, und wo hört die selbst verordnete „Realness“ auf?9 Besonders im deutschen Rap, wo seit Jahren ohnehin das vermeintliche Schreckgespenst vom Gangsta-Rapper mit türkischem oder tunesischem Migrationshintergrund umherstreift und als Paradefigur des integrationsunwilligen Ausländers mit Affinität zur Kriminalität medial ausgeschlachtet wird, findet der Diskurs Zielscheiben. In den letzten Jahren wurde der Prototyp des „G-Rappers“ vor allem vom Berliner Bushido (sowie einer langen Liste seiner Fachkollegen) verkörpert. An anderer Stelle haben wir detaillierter gezeigt, dass die Darstellungen des G-Rap-Genres als symbolischer Bezugspunkt Eingang in eine öffentliche Diskussion findet, die Einwanderung nach Deutschland mit Aspekten jugendlicher Delinquenz und steigender Ge-

sche Studien zu diesem Komplex finden sich in den HipHop-Studies recht gut ausgearbeitet. Auch ist die Kulturgeschichte nach unserem Eindruck mittlerweile über diverse Dokumentationen, Filme, Magazinartikel und Fachzeitschriften recht weit ins Bewusstsein der breiten Öffentlichkeit gelangt. Für deutschen Rap empfehlenswert sind etwa Klein und Friedrich (2003) sowie Loh und Güngör (2002), für die amerikanische Szene bieten sich Toop (2001), Kage (2003) sowie Forman und Neal (2012) an.

${ }^{8}$ Vgl. Marc Dietrich 2012.

${ }^{9}$ Die Realness lässt sich als ein Kulturdispositiv fassen, das zwar in den letzten Jahren an Einfluss verloren hat, im Gangsta-Rap jedoch noch sehr wirksam ist. Es handelt sich dabei um die Forderung nach einer Kongruenz von Leben und Kunst. Rap wird verstanden als Medium der Straßenrealität, die es anhand tatsächlicher biografischer Erfahrungen zu schildern gilt. Bezugspunkt der „Streetales" ist immer wieder der entstammte Sozialraum, das Ghetto oder die Hood, prekäre Räume also, denen Murray Forman (2002) eine Art Beglaubigungsfunktion im Sinne einer „hardcore urban reality" zugeschrieben hat. Es scheint plausibel eher eine inszenierte Realität als Kernstrategie der performativen Praxis anzunehmen, als die Lyrics „für bare Münze“ und eindeutig mit der Person verknüpft zu sehen. 
waltkriminalität vermischt. ${ }^{10}$ Zum Gangsta-Rapper lässt sich zunächst sagen, dass es sich aus unserer Sicht um eine Figur handelt, die eine Projektionsfläche für gesellschaftliche, komplexitätsreduzierende Ordnungsversuche darstellt: Im Anschluss an Moebius und Schroer ${ }^{11}$ erfassen wir Gangsta-Rapper insofern als „Sozialfiguren“. Diese sind zu verstehen als „Typisierungen [...], mit denen Ordnung in die Vielfalt der empirischen Erscheinungen gebracht werden soll“ ". ${ }^{12}$ Dieser Ordnungsversuch hat mit einem traditionsreichen Diskurs um die Rolle von Menschen mit Migrationshintergrund zu tun. Im vorliegenden Kontext reicht es auf Folgendes hinzuweisen:

Vermutete Anomietendenzen im Spannungsfeld von „Mehrheits- und Parallelgesellschaft ${ }^{\text {“13 }}$ werden ganz häufig mit dem dekontextualisierten Verweis diskutiert, dass Menschen mit Migrationshintergrund eine eminente Rolle in dieser Entwicklung zukomme. Dieser - tendenziöse - Verweis kann vor dem Hintergrund bereits historischer Zuschreibungen gesehen werden. So beschreibt der Sozialstrukturforscher Rainer Geißler den Zeitraum zu Beginn der 1980er Jahre mit dem Begriff der „Abwehrphase“ der deutschen Integrationspolitik. ${ }^{14}$ Nicht zuletzt unter dem Eindruck der seit 1973 immer wieder aufkeimenden wirtschaftlichen Rezession, welche sich einwanderungspolitisch nicht zuletzt im 1973 verhängten Anwerbestopp äußerte, prägten Neologismen wie der des „Asylanten“ einen Diskurs über „volle Boote“ und „Ausländerfluten“, die über „uns“ hereinbrächen (u. a. m.). Das Sprechen über „Ausländer“ kann dabei auch nicht unbedingt als erfahrungsgesättigt beschrieben werden. ${ }^{15}$ Während die pathologische Darstellung von Migranten im Spiegel der deutschsprachigen Medien sicherlich keine besonders neue Entwicklung darstellt, ${ }^{16}$ vollzieht sich seit einigen Jahren eine wahrnehmbare Zuspitzung entsprechender Ressentiments

\footnotetext{
${ }^{10}$ Vgl. Marc Dietrich und Martin Seeliger 2012.

${ }^{11}$ Vgl. Stephan Moebius und Markus Schroer 2010.

${ }^{12}$ Ebenda, S. 8.

${ }^{13}$ Diese letzte Formulierung gibt den Modus der Thematisierung wieder und unterstellt keineswegs eine derartige sozialtopologische Gliederung der Gesellschaft.

${ }^{14}$ Vgl. Rainer Geißler 2006, S. 235.

${ }^{15}$ Einem Befund Geißlers (2006, S. 246) zufolge konnten darüber hinaus im Jahr 1980 lediglich $15 \%$ der Westdeutschen in Deutschland lebende Ausländer/Ausländerinnen zu ihrem Freundes- oder Bekanntenkreis zählen (2002 waren es mit über $60 \%$ immerhin vier Mal so viele).

${ }^{16}$ Vgl. Rainer Geißler und Horst Pöttker (Hrsg.) 2005.
} 
in der öffentlichen Auseinandersetzung. So ist nach Hartmann in den letzten Jahren eine „deutliche Verschärfung der Debatte über Multikulturalismus und das angebliche ,Scheitern der multikulturellen Gesellschaft" zu beobachten." ${ }^{\text {"17 }}$

Den Anfangspunkt einer Welle derartiger Berichte machte die Anfang 2008 erschienene Ausgabe des Nachrichtenmagazins „Der Spiegel“ mit ihrer Titelstory „Migration der Gewalt. Junge Männer - Die gefährlichste Spezies der Welt. “18 Nachdem Ende 2007 ein Münchener Hauptschulrektor Opfer einer brutalen Attacke zweier Jugendlicher geworden war, ${ }^{19}$ wird dies in der deutschen Medienlandschaft zum Anlass für eine äußert skandalisierende und pauschale Berichterstattung genommen, im Zuge derer (männliche) Jugendliche mit Migrationshintergrund als generell zu Gewaltdelinquenz neigend dargestellt werden. Dass die Zuschreibung entsprechender Eigenschaften an Einwanderer (oder deren Nachkommen) nicht allgemeingültig ist - man spricht nicht von Skandinaviern, Australiern, US-Amerikanern, Chilenen, Japanern, usw. -, sondern auf spezifische Herkünfte abzielt (in aller Regel der arabische Raum oder Osteuropa, d. h. die Staaten der ehemaligen Sowjetunion), kann hier abgelesen werden. Mit Bude kann man die konstruierte Sozialfigur vom (klischeehaften) jungen Deutschen mit türkischem Hintergrund und muslimischer Affinität, wie folgt beschreiben: „Das stereotypische Bild dafür ist der handybenutzende, jeansbehoste, körperstolze deutschtürkische Jungmann, der sich in Berufung auf den Koran um die ,Ehre' seiner unter dem schlechten Einfluss verderbter Emanzipationsvorstellungen stehenden Schwester sorgt. ${ }^{\text {“20 }}$

Interessanterweise werden nach den Gangsta-Rappern nun Rapper mit Migrationshintergrund und muslimischem Glauben Objekt eines „Krisendiskur-

\footnotetext{
${ }^{17}$ Eddi Hartmann 2008, S. 503.

${ }^{18}$ Der Artikel weist eindeutig essentialistische Aussagen und tendenziöse Argumentation auf: http: //www.spiegel.de/spiegel/print/d-55294620.html (05.08.2012). Deutlich wird auch, dass an der skandalisierenden Berichterstattung keineswegs nur Boulevardmedien mitwirken, sondern auch „intellektuelle“ Organe wie die Zeit: „Mit Gürtelschnallen dreschen junge Türken auf einen Polizisten ein“ rückt in einem Dossier über ethnisierte Jugendgewalt (Lebert und Willeke 2008) immerhin an die prominente Stelle einer Zwischenüberschrift, selbstverständlich ohne dass etwaige Motive (wie z. B. möglicherweise auch Affekt, Verteidigung) weiter in Betracht gezogen würden. Vgl. hierzu auch die Beiträge von Sabine Schiffer (S. 121-137) und Nina Kalwa (S. 139-158) in diesem Sammelband.

${ }^{19}$ Vgl. Joachim Käppner 2008.

${ }^{20}$ Heinz Bude 2006, S. 35.
} 
ses“, der den neuen unliebsamen Prototypen vom „islamischen“ oder gar „,islamistischen Rapper" heraufbeschwört - ohne dass so richtig klar ist, wer damit überhaupt gemeint sein könnte bzw. was das eigentlich ist. Die „bewährte“ Sozialfigur gewinnt an Attraktivität, weil sie mit einer Zusatz-Semantik aufgeladen werden kann, die latent schon mitgeführt wurde. Der Rapper gerät nicht nur zur Personifikation des bildungsunwilligen, gewaltbereiten und parallelgesellschaftsfixierten Menschen mit ausländischen Wurzeln, sondern auch zum vermeintlichen Repräsentanten einer Religion, die oft als anachronistisch bis extremistisch wahrgenommen wird. Wir werden zu zeigen versuchen, dass der gesellschaftlich und medial konstituierte Rahmen der Wahrnehmung von Menschen mit Migrationshintergrund nicht nur in die Behandlung von Gangsta-Rap, sondern auch in die Stilisierung des sog. ,islamischen Rappers“ hineinspielt.

Folgendem möchten wir uns zumindest skizzenhaft nähern durch:

- einen Einblick in die amerikanische Rapszene, aufgrund ihrer „Mutterschiffs-Funktion“ für die deutsche Szene. Wir umreißen die Verbandelung von US-Rap mit islamischen/islamistischen Gruppen, die tatsächlich zu so etwas wie politischem islamischem bzw. islamistischem Rap geführt hat. Hierbei berufen wir uns insbesondere auf die Studie des Anthropologen Ted Swedenburg „Islam in the Mix“ (1997);

- einen kompakten Einblick in die deutsche Rapszene, die sich vor dem Hintergrund von Glokalisierungsprozessen ${ }^{21}$ konstituierte und bisweilen analog funktionierende (amerikanisch-stämmige) Sprecherpositionen (z. B. Gangsta-Rapper) zu Tage förderte.

Zuletzt versuchen wir uns an einem Fazit, das der Frage nachgeht, ob Rap für die Integration muslimischer Semantiken eine geeignete Bühne darstellt, und ob islamischer Rap in Deutschland ein soziologisch und medial relevantes Phänomen ausmacht.

\section{Islam und Rap in den USA}

Wenn man deutschen Rap als Produkt oder gar reine Kopie des amerikanischen Modells begreift (was öfters getan wird, aber tatsächlich nur unter Einschränkungen der Fall ist), so ist man geneigt, amerikanische Phänomene und deren

${ }^{21}$ Vgl. Roland Robertson 1998 sowie Malte Friedrich und Gabriele Klein 2003. 
Integration auch in der deutschen Kultur als in ihrer Funktionsweise analog zu verstehen. In manchen Fällen macht dies sogar Sinn. ${ }^{22}$ Was allerdings nicht analoge Durchsetzung gefunden hat, ist die Sprecherposition „islamischer/islamistischer Rapper“.

Soviel sei an dieser Stelle schon gesagt. Die schnelle Rede von Islam(isten)Rap ist auch für den US-Raum nicht unproblematisch: Auseinanderzuhalten sind einerseits US-amerikanische Sprecherpositionen, die sich mit dem Label „PolitRap“ (nicht Gangsta-Rap) identifizieren lassen und an denen sich islam-affine (nicht islamische) bis extremistische Orientierungen ablesen lassen. Zu letzterer Sorte zählen solche Sprecher, die insbesondere in den 1980er Jahren und frühen 1990er Jahren als Aktivisten der so genannten 5 Percenter präsent waren. Hierbei handelt es sich um eine Splittergruppe der ohnehin schon recht autark vom dogmatischen Islam agierenden Nation of Islam (NOI).

Die zweite Gruppe von Sprechern arbeitet mit Referenzen an den Islam, ist aber programmatisch nicht so aufgestellt, dass man hier tatsächlich von islamischem oder islamistischem Rap sprechen könnte (z. B. bei den mittlerweile aufgelösten Fugees). Widmen wir uns also den 5 Percentern, die nicht nur in ihrer musikalischen Repräsentation einen relevanten Gegenstand abgeben, sondern sicherlich auch religionssoziologisch von Interesse wären. Hier lässt sich noch ein Forschungsdesiderat ausmachen.

Die folgenden Ausführungen zu diesem wenig erforschten Gegenstand basieren auf einem Essay, den der Anthropologe Ted Swedenburg bereits 1997 verfasst hat. ${ }^{23} \mathrm{Da}$ einige Rapper bis heute aktiv sind und oder gehört werden, haben die Erkenntnisse durchaus noch Gültigkeit.

\footnotetext{
22 So kann mit Recht davon gesprochen werden, dass der deutsche Gangsta-Rapper sich etablieren konnte und ähnlich funktioniert wie sein amerikanisches Pendant, weil gewisse Diskursverschränkungen in den USA den deutschen nicht unähnlich sind und in beiden Fällen eine Außenseiterposition reklamiert werden kann. Der afroamerikanische Gangsta-Rapper inszeniert sich als „bad man“, der sich in der Figur des "Niggaz" manifestiert, so wie sich der deutsche Gangsta-Rapper mit Migrationshintergrund als „Kanacken-Rapper“ (oder wie es neuerdings heißt „Azzlack“) präsentieren kann.

${ }^{23}$ Vgl. http://comp.uark.edu/ tsweden/5per.html (05.08.2012).
} 


\subsection{Nation of Islam und 5 Percenters}

Nicht an der Oberfläche, wohl aber omnipräsent sind NOI-Verweise oder Referenzen an die 5 Percenter in US-Rap-Texten und Performances. Die in Europa wenig bekannte und unkonventionelle religiöse Gruppe hatte massiven Einfluss auf die Szene in den USA - von Gangstarr bis zum Wu-Tang-Clan.

Bei der 5 Percent Nation bzw. der Nation of Gods and Earths, ${ }^{24}$ wie die Eingeweihten häufig sagen, handelt es sich um eine religiöse Gemeinschaft, die nur wenig mit dem fundamentalistischem Islam teilt, aber große Gemeinsamkeiten mit der Nation of Islam Louis Farrakhans hat. Gegründet wurden die 5 Percenter von Clarence 13X, der in den 1950er Jahren zunächst einem Harlemer NOITempel beitrat, der zu dieser Zeit von Malcolm X geführt wurde. In den 1960er Jahre distanzierte sich Clarence $13 X$ von der NOI. Farrakhans Nation nahm an, dass 1930 in Detroit mit Master Farad Muhammad Gott erschienen sei. ${ }^{25} \mathrm{Cla}$ rence $13 X$ vollzog den Split mit dem Verweis auf Farads Hautfarbe: Farad sei nicht schwarz, sondern von eher heller Hautfarbe gewesen (er hatte vermutlich asiatische Wurzeln). Dies - so Clarence - kollidiere mit der einstigen NOIAnnahme, der zufolge Allah nur schwarz sein könne. An dieser Überzeugung wollte Clarence festhalten.

Clarence' Ideologie war mit der Überzeugung verbunden, dass prinzipiell in jedem schwarzen Mann Göttliches stecke. Dieses Potential gelte es durch Studium und intensive Reflexion zu entwickeln. Der Prediger verließ die NOI und nahm eine Reihe von Anhängern mit sich. Fortan wurde er Father Allah genannt und verbreitete seine religiöse Message auf den Straßen Harlems - eine Praxis, die von den traditionellen NOI-Seminaren im Sinne eines „question-and-answer format ${ }^{\text {“26 }}$ abwich und so wohl auch Gehör bei straßenaffinen Vertretern der späteren HipHop-Community fand.

\subsection{Das Weltbild der 5 Percenter}

Der Name der 5 Percenter rührt von einem durch Wissenshierarchien gekennzeichneten Weltbild her: Die vielleicht elementarste Überzeugung besteht in

\footnotetext{
${ }^{24}$ Vgl. ebenda, S. 1.

${ }^{25}$ Vgl. ebenda, S. 3.

${ }^{26}$ Vgl. ebenda, S. 4.
} 
der Annahme, dass fünfundachtzig Prozent der Bevölkerung eine verblendete und unwissende Existenz führt. Zehn weitere Prozent sind wissend, setzen diese Wissensbestände jedoch zum eigenen Vorteil und unter Inkaufnahme von Ausbeutung der Übrigen ein. Unter ihnen befinden sich maßgeblich Weiße als „bloodsuckers of the poor“ ${ }^{27}$ Lediglich 5 Prozent der Menschheit sind prädestinierte Wissende, die Kenntnis vom Lauf der Dinge auf Erden besitzen und gewissermaßen eine Meta-Perspektive beanspruchen können.

Die Hauptbezugspunkte der gläubigen 5 Percenter sind die von Father Allah entwickelten so genannten Supreme Mathematics und Supreme Alphabets, ${ }^{28}$ die auch nach seiner Ermordung 1969 weitergegeben wurden und sich in anderen Großstädten - besonders unter jungen Afroamerikanern - der USA zu etablieren wussten. Es handelt sich hierbei weniger um feste Glaubenssätze als vielmehr spirituelle Annahmen, die im Eigenstudium zu durchdringen sind. Die Supreme Mathematics sind im Grunde als Numerologie mit Welterklärungsanspruch zu verstehen. Eine prominente Rolle in der Lehre, die das Verhältnis des Menschen zum Universum zu beschreiben sucht, ${ }^{29}$ spielt die Nummer „ $7^{\prime 3} .{ }^{30}$

Seven is the number of perfection. It stands for the seventh letter of the alphabet, G, and for God. (Five Percenters originated the homeboy expression, "sup (what's up), G?", originally "G” stood for God, not gangsta.). According to Jah-Z Allah, keeper of the $5 \%$ website, "The God sees in cyles of 7 colors of the rainbow and hears in 7 cycles of notes on the musical scale." According to Farad's lessons, the Original (Black) Man has 7, 5 ounces of brain, the (white) devil, only 6 ounces (Farad, Lost Found Muslim Lesson No. 2). The flag of the Five Percenters, known as the Universal Flag of Islam, contains a 7 (symbol of God) surrounded by a crescent moon (signifying the black woman) and a star (signifying the child). ${ }^{31}$

Typisch für 5 Percenter ist auch ein gründlicher, aber eigenwilliger Umgang mit Sprache. Man geht Wortteilen auf den Grund („breakin’ it down“) und versucht auf diese Art neue Deutungen von Wörtern zu erschließen. ${ }^{32}$ Dieses Cha-

\footnotetext{
${ }^{27}$ Ebenda.

${ }^{28}$ Vgl. ebenda, S. 5.

${ }^{29} \mathrm{Vgl}$. ebenda.

${ }^{30} \mathrm{Vgl}$. das Symbol unter http://api.ning.com/files/-VCqNhXiur-XLob0ipFvknLsE6CpNbjkBOMjQDf6X9TcP6QkQ WkOkjobrfhURLGLn7X90sAwLcl7aF6o0N9J3HG9WRSYE6CI/UniversalFlag.gif (05.08.2012).

${ }^{31}$ Ebenda.

${ }^{32}$ Vgl. ebenda, S. 7.
} 
rakteristikum des kreativen Umgangs mit Sprache teilen Rap ${ }^{33}$ und die 5 Percenter, so wie auch das „Predigen“ auf der Straße eine gewisse Gemeinsamkeit mit dem Rappen „an der Straßenecke“ darstellt. Viele Wort-Wendungen wie etwa „droppin science“ oder auch das obligatorische „peace“, das einem Vorgänger der 5 Percenter, dem so genannten Moorish Science Temple, entlehnt ist, sind in das HipHop-Vokabular eingedrungen und haben ihren originalen Bedeutungsgehalt im kollektiven Gedächtnis der amerikanischen Rapkultur eingebüßt. ${ }^{34}$

Nach der Lehre der 5 Percenter zu leben bedeutet primär ein Eigenstudium nach wenigen orientierenden Leitgedanken zu verfolgen. Die Ausbildung des „knowledge of self“, die selbstbestimmte Aneignung universellen Wissens, ist das Ziel. Jeder Einzelne der erlesenen Wissenselite hat sich selbst durch Bildung und Studium der Welt bzw. des Menschen seinen Status erarbeitet (so die Überzeugung). Der 5 Percenter hat sich dann zum „god“ empor gearbeitet - eine Möglichkeit, die prinzipiell jedem disziplinierten, wissbegierigen und vorzugsweise schwarzem Menschen aus Sicht der 5 Percenter offen steht. Gott ist also keine Figur im monotheistischen Sinne, sondern etwas, das im Menschen selbst erweckt werden kann und somit konzeptuell dem Pantheismus zugehörig ist.

\subsection{Lebenspraxis}

Wie Swedenburg betont, sprechen sich einige 5 Percenter in islamischer Manier gegen den Konsum von Alkohol und Drogen aus. Einige Rapper widersprechen dieser asketischen Haltung, indem sie auf die Ansicht verweisen, dass ein jeder Gott seines eigenen Universums sei und so ein Stück weit nach seinen eigenen Regeln leben solle. ${ }^{35}$

Weitere verbreitete Elemente des Glaubens sind die folgenden, die prominente Rapper in der Lebenspraxis häufig selektiv-, nicht- oder konträr ausgelegt haben:

\footnotetext{
${ }^{33}$ Die 5-Percenter-Elemente im Rap lassen sich aber auch auf der Oberfläche finden: Der Rapper Lord famar (Brand Nubian) etwa releaste ein „5\%-Album“, das konsequenterweise das Symbol der religiösen Strömung schon auf dem Cover trägt. Vgl. unter http://www.bing.com/images/search?q=lord+ jamar + the $+5+$ percent + album\&view $=$ detail\&id $=$ D810C695E80B7FAC808AC15456310BEEA146396B\&FORM $=$ IDFRIR (05.08.2012).

${ }^{34}$ Vgl. ebenda, S. 6.

${ }^{35}$ Vgl. ebenda, S. 7.
} 
- Die Rolle der Frau ist tendenziell eine dem Mann untergeordnete: „Just as earth resolves around the sun, woman is subordinate to man. ${ }^{36}$ Grundsätzlich kann die Frau nach Maßgabe der 5 Percenter-Lehre nicht das Stadium der Perfektion erreichen (,7“), sondern lediglich zu daran angrenzenden Stufen (maximal „6") hervordringen. Ein komplettes „knowledge of self“ bleibt also eine männliche Domäne. ${ }^{37}$

- Weiße Menschen haben ebenfalls einen prekären Status inne: Der Überzeugung folgend, dass der weiße Mann das 6.000 Jahre alte Produkt eines bösen Wissenschaftlers mit Namen Yacub ist (daher auch die bisweilen anzutreffende Wendung vom „white devil"), haben Weiße eine antagonistische Rolle innerhalb des orthodoxen Weltbildes inne. ${ }^{38}$

\subsection{Schlaglichter auf Inszenierungen im US-Rap}

Der Spagat zwischen autonomer Lebensführung und fundamentalistischer Glaubenspraxis führt nicht selten zu widersprüchlichen Performances. Bei Big Daddy Kane etwa - einem bekennenden 5 Percenter mit Klassikerstatus in der Rapszene - dokumentiert sich eine recht hybride Inszenierung, die Nacktfotos im Playgirl Magazin ${ }^{39}$ ebenso zu integrieren versuchte wie die Ode an den Hedonismus im 1989er Hitvideo zu „Smooth Operator“ ${ }^{40}$ Dort inszeniert sich Kane als gewinnsüchtiger Player und versierter Verführer, der dazu den „VorzeigeOriginal-Black-Man“ im Sinne eines afroamerikanischen Königs zu geben versucht. ${ }^{41}$ Garniert mit 5 Percenter-Floskeln („word is bond“, „peace“) rief der selbst ernannte King Asiatic Nobody's Equal auch Kritiker aus den eigenen Reihen auf den Plan.

Ein anderer interessanter Fall ist der einstige NOI-Repräsentant und heutige Agnostiker Paris. Fast das gesamte musikalische Schaffen des studierten Wirt-

\footnotetext{
${ }^{36}$ Ebenda.

${ }^{37}$ Vgl. ebenda.

${ }^{38}$ Vgl. ebenda, S. 8.

${ }^{39}$ Vgl. unter: http://www.blackdogue.net/Playgirl2/BigDaddyKane/BigDaddyKane.html (05.08.2012).

${ }^{40}$ Vgl. unter: http://www.youtube.com/watch?v=8g5bjSUysQA (05.08.2012).

${ }^{41}$ Eine detaillierte Video- und Textanalyse findet sich in der laufenden Dissertation von Marc Dietrich „Rapresent what? Zur Inszenierung von Ethnizität, Authentizität und sozialer Differenz im RapVideo".
} 
schaftswissenschaftlers und Teilzeit-Stock-Brokers konzentriert sich auf Themen wie „Polizeigewalt gegen Afroamerikaner“, „Diskriminierung“, „Rassismus“ sowie „US-amerikanischer Imperialismus“. Schon der erste Schritt auf die popkulturelle Bühne mit „The devil made me do it“ zeigte den Rapper als problack-Aktivisten (,pro black and it ain't no joke“). ${ }^{42}$ Das Video zeigt einen rassistischen weißen Polizisten, der u. a. die (angedeutete) Vergewaltigung einer schwarzen Frau unternimmt und bei allen Taten von einem bizarren „UncleSam-Verschnitt“ begleitet wird. ${ }^{43}$ Jene Version des Uncle Sam wird hier zweifelsohne zur Ikone der „white supremacy“ und damit zum „white devil“ stilisiert. Die Raplines sind unschwer erkennbar mit NOI-Elementen gespickt: Revolution ain't never been simple; Following the path from Allah for know just; Build your brain and we'll soon make progress; Paid your dues, don't snooze or lose; That came with the masterplan that got you; So know who's opposed to the dominant dark skin; Food for thought as a law for the brother man.

Im Auftrage Allahs gilt es, sich mit Wissen für die Revolution zu wappnen: Dabei muss klar sein, wer der Feind ist, nämlich derjenige, der „opposed to the dominant black skin" ist und in Form des White Devil/Uncle Sam auf der Bildebene eingeführt wird. Ton und Aussage der Musik von Paris kreisen bis heute um solche Antagonismen zwischen weißer Hegemonie und schwarzer Marginalisierung. Die 5 Percenter-Rhetorik tritt im späteren Werk durch den Wechsel zum Agnostizismus zurück und konzentriert sich stärker auf eine Kulturkritik, die Amerika und letztendlich auch den sog. weißen Mann stets als Bedrohung für andere Kulturen und Religionen führt. Dabei begibt sich Paris in seiner Ikonographie zum Album mit dem provokativen Titel „Sonic Jihad“, dass nur wenige Jahre nach dem 11. September 2001 herausgebracht wurde, in die Nähe von Dschihadisten. ${ }^{44}$

Fassen wir vorläufig zusammen:

Im US-Rap findet sich eine lange Tradition von Rappern mit Bindung zum Islam. Zwei prominente Figuren haben wir gestreift. Darüber hinaus existiert

\footnotetext{
${ }^{42}$ Vgl. unter: http://www.lyricsmode.com/lyrics/g/golden_earring/the_devil_made_me_do_it.html (05.08.2012).

${ }^{43}$ Vgl. unter: http://www.youtube.com/watch?v=hKRAFPHD8W4 (05.08.2012).

${ }^{44}$ Das Albumcover zeigte das Bild von einem Flugzeug, das zielgerichtet auf das Weiße Haus zuflog. Vgl. http://www.guerrillafunk.com/paris/sonic jihad/ (05.08.2012).
} 
eine große Anzahl von Rappern, die hier nicht explizit behandelt werden konnten, Rapper, die auch aktuell in allen Ohren sind. Freeway und Mos Def pflegen Referenzen an den Islam vorzunehmen, ohne dabei inszenatorisch einer dezidiert religiösen Programmatik verschrieben zu sein. Man ist Muslim, ohne diese Eigenschaft in extenso für die Performance einzusetzen. Auf der anderen Seite sind es Polit-Rapper wie Paris (oder Dead Prez, Brand Nubian, Public Enemy und The Coup), die die historische Marginalisierung schwarzer Bürger mit Black Panther- und NOI-gefärbten Inhalten zusammenbringen. Hierbei handelt es sich vornehmlich um Rapper, die in den 1980er- und 1990er-Jahren relevant waren. In dieser Zeit finden sich deutlich mehr Referenzen an die NOI oder eben die 5 Percenter als in der Gegenwart.

Für Inspiration sorgen dabei also insbesondere islamische Strömungen, die die Rolle schwarzer Akteure aufwerten. Die Attraktivität der Verbindung von problack-orientierter Religion und Rap liegt sicherlich auch in der Ursprungserzählung von Rap als Stimme der Straße und „Black CNN“ (Chuck D). Rap wird hier als Gegenkultur interpretiert, die die marginalisierte Position des „Schwarzen Mannes“ mit Verweis auf eine rassistisch motivierte, „weiße“ Kultur-Hegemonie reflektiert. Afrozentrische Emanzipationsversuche, die sicher nicht zufällig in der Reagan- ̈̈ra aufkeimten und sich wie geschildert auch auf religiös-ideologische Versicherungen berufen konnten, vermochten sich im Medium einer als „schwarz" gedeuteten ästhetischen Praxis zu kanalisieren. ${ }^{45}$

Dieses Phänomen ist in der Retrospektive jedoch eher von kurzfristiger Natur gewesen. Schaut man auf den Status quo der amerikanischen Rapszene, so scheint diese inhaltlich wie inszenatorisch-ästhetisch weitestgehend im postmodernen „anything goes“ aufgegangen zu sein. Aufstrebende Künstler wie die Gruppe ODD Future um Tyler the Creator ${ }^{46}$ und der Rapper Spaceghost Purrp ${ }^{47}$ spielen mit religiösen bzw. satanistischen Symbolen genauso wie mit fiktiven Gewaltverbrechen oder der Integration absurder mythologischer Szenerien, ohne dass eine dezidiert politische oder religiöse Verortung in übergreifender Form ablesbar wäre.

\footnotetext{
${ }^{45}$ Vgl. dazu Tricia Rose 2008, S. 42-44.

${ }^{46}$ Vgl. Stephan Szillus 2012, S. 20-26.

${ }^{47}$ Vgl. Zainab Jama 2012, S. 29.
} 
Es mag auch mit der Tatsache zu tun haben, dass die USA mittlerweile und erneut von einem schwarzen Demokraten regiert werden (Stand November 2012), dass die Lyrics über eine weiße Übermacht und die Notwendigkeit schwarzen Aufbegehrens weniger Durchschlagskraft entfalten. Die Sprecherposition „islam-affiner Polit-Rapper" ist wohl auch aus diesem Grunde nicht mehr sonderlich „in Mode“.

\section{Rap und Islam in Deutschland}

Wenn deutscher Rap sich durch die so genannte "Glokalisierung" ${ }^{48}$ - das heißt in diesem Fall die lokal gefärbte Anverwandlung und Adaption von medial verbreiteten US-Inszenierungen - herausgebildet hat, so könnte man annehmen, dass so etwas wie islamischer Rap auch in der deutschen Szene Etablierung gefunden hat. Schließlich gibt es ja auch deutschen Gangsta-Rap. In Deutschland sind die Diskussion und auch die „popkulturelle Realität“ allerdings etwas anders gelagert als in den USA. Politischer Rap ist gegenwärtig kaum auszumachen. Eine nähere Betrachtung des Diskurses muss feststellen, dass der mit dem Islam verbandelte Rapper auch eher in der Sprecherposition des Gangsta-Rappers oder zumindest im straßenorientierten Rapper vermutet wird. Der politische Rap wurde in Deutschland jedoch lange Zeit eher von Nicht-Muslimen wie dem Kulturmitbegründer Torch und seiner Gruppe Advanced Chemistry praktiziert, bevor linke Untergrundgruppen wie Anarchist Academy auftauchten. Mit G-Rap hat dies nichts zu tun. Mit etwas Mut zur großzügigen Kategorisierung kann man aktuell Rapper wie den iranisch-stämmigen Fard noch am ehesten als politisch bezeichnen. Martin Seeliger erkannte Klassenkampfmotive - und damit politischen Gehalt - bei Nate $57 .{ }^{49}$ Islami(sti)sche Inhalte finden sich hier aber ebenfalls nicht. Angesprochen auf den Status und die Präsenz von islamischem Rap in Deutschland bemerkt der Chefredakteur des europaweit einflussreichsten Rap-Magazins fuice, Stephan Szillus, in einem privaten Gespräch, dass es so etwas wie islamischen Rap in Deutschland eigentlich nicht gebe.

\footnotetext{
${ }^{48}$ Vergleiche zum Begriff im allgemeinen Robertson (1998) und hiphop-spezifisch Klein und Friedrich (2003, S. 85-113).

${ }^{49}$ Vgl. Martin Seeliger 2012.
} 
Kommen wir noch einmal auf den Gangsta-Rapper zurück: Gangsta-Rap in Deutschland erfüllt soziologisch betrachtet eine ganz ähnliche Funktion wie in den USA. Er wird in der Öffentlichkeit tendenziell als Plattform für gewaltbereite junge Erwachsene mit defizitärem Bildungs- und oft auch Migrationshintergrund gesehen. Die Genre-Größen fungieren häufig in Talkshows, Tageszeitungen und Fernsehreportagen als Personifizierung des gesellschaftlich Dysfunktionalen, als Projektionsflächen befürchteter anomischer Auswüchse. Die von den USA inspirierten Lyrics über das harte Leben auf der Straße im Spannungsfeld von Aufstiegsfantasien, zur Schau getragenem Outlaw-Stolz und Gangsta-Tales bringen Stilisierungen hervor, die analog zu amerikanischen Brennpunkten funktionieren: Marginalisierte Gegenden wie Berlin-Neukölln oder die Frankfurter Nordweststadt werden zu mythisch besetzten Orten, die Compton (Los Angeles) oder der Bronx (New York) in kaum etwas nachstehen. Ähnliches lässt sich über die Funktionsweise der Textsubjekte behaupten: Der „Nigga" als kriminell aufbegehrendes, gewaltbereites und zentrales Subjekt des amerikanischen Gangsta-Rap wie ihn N.W.A. kreiert haben, kann als inszenatorisches Analogon zum Kanacken-Rapper, zum „Azzlack“ (um mit dem Genrehelden Haftbefehl zu sprechen) gesehen werden. ${ }^{50}$ Dabei rührt die gesellschaftliche Abwehr des Gangsta-Rappers mitunter von Ressentiments respektive der Hautfarbe (im Falle der USA: Race) bzw. dem migrantischen Hintergrund (Deutschland) her. Hier greift die Glokalisierung also auf interessante Weise und mit soziologisch relevanten Effekten.

Es sind unsere Thesen, dass der Gangsta-Rapper (1) aufgrund seiner provokanten und Ängste um eine vermeintliche Parallelgesellschaft schürende Inszenierung kritisiert und (2) besonders deshalb der islamistischen Propaganda verdächtigt wird, weil sich Menschen mit türkischem oder tunesischem Hintergrund in diesem Feld betätigen - Bevölkerungsgruppen also, denen man eine Affinität zu islamistischen Haltung und Integrationsverweigerung ohnehin zu unterstellen geneigt ist.

${ }^{50}$ Vgl. Marc Dietrich 2012. 


\subsection{Gangsta-Rapper als Islamisten? Deso Dogg und andere}

Wenn der Gangsta-Rapper also als eine Sozialfigur mit komplexitätsreduzierender Funktion („Ordnung herstellen“) zu denken ist, an der die kampflustige Diskussion um jugendliche Migranten mit Kriminalitätsneigung und Bildungsunwilligkeit einen geeigneten Sandsack findet, dann zielt der Diskurs um islamische/islamistische Akteure mit dem Rapper Deso Dogg (bürgerlicher Name: Denis Mamadou Cuspert) auf eine Figur, die scheinbar noch eine weitere beängstigende Eigenschaft aufweist: Er ist nicht nur (Ex)-Gangsta-Rapper, sondern auch noch Salafist. In einem medial mitkreierten Klima der Angst vor „Überfremdung" und der polarisierenden Debatte um die Zugehörigkeit des Islams zu Deutschland findet sich hier scheinbar die Personifizierung des gesellschaftlichen Übels. Diese Diskussion ist schnell zu beruhigen: Denis Cuspert ist zwar sicherlich ein eingespannter Seelenfänger und Verbreiter fragwürdiger islamistischer Inhalte, aber eben kein Rapper. Nicht mehr. Bei YouTube findet man einen Aufruf an die Fans, seine Musik zu vernichten, gespickt mit Beteuerungen, das Musikerleben habe ihm übel mitgespielt, er wolle warnen vor diesem „falschen Weg“, der mit dem Glauben nicht zu vereinbaren sei. ${ }^{51}$

Dass die Medien dies ignorieren und trotzdem quer durch alle Magazine und Formate vom Hass- und oder Islamisten-Rapper die Rede ist, ${ }^{52}$ spricht weniger für die journalistische Gründlichkeit, als für das zweifelhafte Auffinden einer enorm anschlussfähigen Semantik, die sich in eben der Sozialfigur des Salafisten-Gangsta-Rappers reflektiert.

\footnotetext{
${ }^{51} \mathrm{Vgl}$. http://www.youtube.com/watch?v=i58F-mCTDDY\&feature= related (05.08.2012).

${ }^{52}$ Dies dokumentiert sich insbesondere bei Boulevardblättern wie der $B Z$ http://www.bz-berlin.de/ archiv/gesuchter-hass-rapper-untergetaucht-article1497573.html (05.08.2012). Aber auch der Focus hebt sich da nicht wesentlich ab: http://www.focus.de/politik/deutschland/ex-rapper-deso-dogg-singt-loblied-auf-bin-laden_ aid_642789.html (05.08.2012). Die Bezeichnung „Rapper“ oder der Rapname in Kombination mit der Betätigung im Salafisten-Feld ist stets präsent. Grundsätzlich würde es reichen, die vormalige Beschäftigung von Abou Maleeq (so der angenommene Name) einmalig zu nennen. Stattdessen evoziert schon die Überschrift „Deso Dogg singt Loblied auf Bin Laden“ einen Nexus von Raptum und extremistischer Orientierung.
} 


\section{Fazit: Rap als Bühne oder kulturelle Praxis für islamische und islamistische Akteure?}

Zweifelsohne ist die (sub-)kulturelle, ästhetische Praxis des Rap ein Betätigungsfeld für Akteure mit ganz verschiedenen Lebensorientierungen und Überzeugungssystemen. Artikuliert wird so ziemlich alles. Es wurde grundsätzlich auch schon über alles gerappt: Banküberfälle, gescheiterte Beziehungen, Schussverletzungen, Orgasmen, die Freude am heterosexuellen (und neuerdings auch homosexuellen) Sex, Liebe, Hass, Lokal- und Nationalpatriotismus, Verabscheuung der Heimat. Sogar über Fußballspiele (Blumentopf) und Würstchenbuden wurde schon gerappt (Imbiss Bronko). Rap als ökonomisch wenig voraussetzungsvolle Praxis $^{53}$ - es genügt im Grunde ein Laptop und ein Mikrofon - mag eine Menge von potentiellen Aktivisten anziehen, die die Musik nicht nur aufgrund der „Hipness“, sondern eben auch zu Versuchszwecken frequentieren. Die Heterogenität und Polyphonie ästhetischer Zugänge hat wohl auch mit der kreative Potentiale erschließenden Produktionstechnik, dem Sampling zu tun, die zwar nicht von Rappern erfunden, aber doch am intensivsten genutzt wurde. ${ }^{54}$ Bei der Produktion von Beats kann man Stücke sampeln und so etwa ein Instrumental mit klassischer Musik (z. B. bei Die Firma, die bei „Das neue Jahrtausend“ ein Sample aus Mozarts 40. Sinfonie nutzt) basteln oder aber ein Instrumental stricken, das auf dem hydraulischen Geräusch von hochfahrenden Autos (so genannten „Lowridern“) basiert (Xzibit „Front 2 Back“). Auch Vocal-Samples gehören zum ästhetischen Inventar. Ein Ausschnitt aus einer Martin-LutherKing-Rede (z. B. Common „A Dream“) ist dabei genauso integrierbar wie ein Kinderchor (fay- $Z$ „Hard knock life“ mit einem Sample aus dem Musical „Annie“). Die Qualität der Anverwandlung der Ursprungsquelle ist dabei von Fall zu Fall zu untersuchen und mit der lyrischen und gegebenenfalls visuellen Performan-

\footnotetext{
${ }^{53}$ Dazu Klein und Friedrich 2003, S. 188, in Bourdieuscher Terminologie: „Während z. B. in der Techno-Szene die Raver über ein gewisses $\mathrm{Ma}$ an ökonomischem Kapital verfügen müssen, um überhaupt an den entsprechenden Events, den Raves und Parties teilnehmen zu können, spielt in den lokalen HipHop-Szenen das ökonomische Kapital, das den globalen Markt der Rap-Musik beherrscht, eine vergleichsweise geringe Rolle. Szenetypisch und positionsanzeigend ist primär also das kulturelle Kapital in Form von szenespezifischem Wissen oder Fähigkeiten in der jeweiligen Disziplin."

${ }^{54}$ Vgl. Lothar Mikos 2003, S. $73 \mathrm{ff}$.
} 
ce in Beziehung zu setzen. Im vorliegenden Kontext muss man festhalten: Ein Zitat aus dem Koran oder ein islamisches Sprachspiel macht noch keine islamische Rap-Performance. Niemand würde beispielsweise die Rap-Gruppe Die Firma aufgrund ihrer massiven Bibelreferenzen für eine christliche Band halten. Ebenso wenig war die Performance von Pussy Riot im Sommer 2012 in einer orthodoxen Moskauer Kathedrale grundsätzlich religiös motiviert, obwohl deren Kritik sich auch auf die Orthodoxe Russische Kirche bezog. ${ }^{55}$

Feststellen lässt sich zudem, dass islam-affine Rapsongs nur sehr vereinzelt auftreten - so wie auch christliche Rapsongs kaum zu hören sind. Über die Gründe können wir an dieser Stelle nur spekulieren: Es könnte damit zusammenhängen, dass Muslime ihren Glauben nicht mit dem verankerten Symbolund Erzählungs-Kosmos des Rap zusammenbringen möchten. Für diese Vermutung sprechen auch die Resultate einer Schwerpunktreportage, die auf ZDFNeo lief. ${ }^{56}$ Nachgegangen wurde der Frage, ob es in Deutschland islamischen bzw. islamistischen Rap gebe. Verschiedene Rapper (Deso Dogg, Manuellsen, Alpa Gun und PA Sports) mit islamischem Hintergrund wurden befragt, wie sie es mit ihrer musikalischen Praxis und der Zugehörigkeit zum Islam halten.

Im Falle Deso Dogg ist es so, dass Musik als etwas betrachtet wird, das gemäß der Glaubensauslegung nicht praktiziert werden kann - Musik als Entertainment sieht der Koran in dieser Lesart nicht vor. Musik ist haram, also Sünde. Etwas anders argumentieren die Rapper Alpa Gun, PA Sports und Manuellsen. Sie plädieren dafür, Religion als Teil des Privatlebens zu betrachten und nicht missionarisch für die Rap-Performance zu instrumentalisieren. Rap sei schlicht die falsche Bühne für den Glauben. Rap als Praxis, die oft den stilisierten Rausch, Promiskuität und Grenzüberschreitung zur Inszenierung einsetzt, mag nicht als ideale Plattform für die religiöse Orientierung gesehen werden.

Extremistische Vertreter sind sich der inkludierenden Wirkung von Rapmusik und dem Einfluss seiner populären Figuren dennoch wohl bewusst: Pierre Vogel konnte Deso Dogg bereits „bekehren“, mit Gangsta-Rappern wie Bushido und

\footnotetext{
${ }^{55}$ Vgl. unter: http://www.fr-online.de/meinung/analyse-zu-pussy-riot-die-kirche-hat-das-nachsehen, 1472602, 16763 688.html (05.08.2012).

${ }^{56}$ Vgl. unter: http://www.zdf.de/ZDFmediathek/hauptnavigation/startseite\#/beitrag/video/1285846/Wild-Germany--Islamischer-Rap (05.08.2012).
} 
Massiv liebäugelte er auch bereits. ${ }^{57}$ Es scheint zudem so, dass Religion und Rap in Deutschland insgesamt kaum in der Lage sind, Verschränkungen im breitentauglichen Sinne einzugehen. Dies mag damit zusammenhängen, dass die oft zirkulierenden, aufregenden Semantiken der lustvollen Grenzüberschreitung, der Eskapismusfantasien und der Zelebrierung von finanziellem Wohlstand, nicht gerade klassische Domänen oder Desiderate der religiösen Lehren sind. Religion scheint für das zeitgenössische Publikum eher das Gegenstück zu allem zu sein, was Rap (und Pop) interessant macht. Es handelt sich letzten Endes also um eine „Aversion“, eine Vermeidungsstrategie, die auf Seite der Produktionsakteure wie auf der Rezeptionsseite vorzufinden ist: Die einen halten ihren Glauben aus verschiedenen Gründen aus dem musikalischen Schaffen heraus, während die anderen offenbar froh sind, von religiösen Inhalten "verschont“ zu bleiben. ${ }^{58}$

Schlussendlich stellen wir fest, dass es sich beim islamischen oder islamistischen Rapper eher um eine Fiktion handelt, die der Diskurs in Deutschland zur Ordnungsstiftung und Kanalisierung latenter Ängste konstruiert hat. Was in der amerikanischen Szene durchaus als medial und soziologisch relevantes Phänomen auffindbar ist und vor dem Hintergrund des skizzierten Diskurses und anderen gesellschaftlichen Konditionen emergierte, kann in diesem Maßstab für die deutsche Szene nicht konstatiert werden. Dass es irgendwo auf YouTube oder in anderen Foren Rapper gibt, die sich an islamischem Rap oder extremistischen Varianten versuchen, ist nicht auszuschließen. Ob dies ein soziologisch relevantes Phänomen darstellt oder einer intensiven Mediendebatte zugeführt werden sollte, ist aber mehr als fraglich.

\footnotetext{
${ }^{57}$ Vgl. unter: http://www.youtube.com/watch?v=zpLH7dpabZc\&feature= related (05.08.2012).

${ }^{58}$ Dass es Produktionen gibt, die sich auch mit misslungener Integration, Diskriminierungen sowie Islamophobie in Deutschland kritisch beschäftigen und die Mehrheitsgesellschaft in die Pflicht nehmen (z. B. Massiv - Ghettolied, Advanced Chemistry - Operation Artikel 3, Automatikk - Vaterstaat, Bushido - Sonnenbank Flavour, Ahmet Gündüz - Fresh Familee) soll nicht bestritten werden. Die erwähnten Tracks fallen allerdings überwiegend in die Jahrtausendwende, bekamen zusätzliche Nahrung durch 9/11, gingen aber in den Folgejahren in die allgemeine Gesellschaftskritik auf. Rückblickend sind sie in ihrer religionsbezogenen Qualität eher als verstreute Phänomene, keineswegs aber als stabile Entwicklungen zu betrachten.
} 


\section{Literatur}

Bude, Heinz, Gläubig - Ungläubig, in: Lessenich, Stephan; Nullmeier, Frank (Hrsg.), Deutschland. Eine gespaltene Gesellschaft, in: Bundeszentrale für politische Bildung, Bonn 2006, S. 295-313.

Dietrich, Marc, Von Miami zum Ruhrpott. Analyse von Gangsta-Rap-Performances in den USA und Deutschland, in: Dietrich, Marc und Seeliger, Martin (Hrsg.), Deutscher Gangsta-Rap. Sozial- und kulturwissenschaftliche Beiträge zu einem Pop-Phänomen, Bielefeld 2012.

Forman, Murray, The Hood comes first. Race, Space, and Place in Rap and Hip Hop. Middletown, Connecticut 2002.

Forman, Murray und Neal, Mark-Anthony, That's the joint. The HipHop Studies reader. Second Edition, New York 2012.

Friedrich, Malte und Klein, Gabriele, Is this real? Die Kultur des HipHop, Frankfurt a. M. 2003.

Geißler, Rainer, Die Sozialstruktur der Bundesrepublik, Wiesbaden 2006.

Geißler, Rainer und Pöttker, Horst (Hrsg.), Massenmedien und die Integration ethnischer Minderheiten in Deutschland. Problemaufriss, Forschungsstand, Bibliographie, Bielefeld 2005.

Hartmann, Eddie, Die „Banlieue-Krise“ und die émeutes urbaines. Soziologische Perspektiven auf einen gesellschaftlichen Konflikt, in: Berliner Journal für Soziologie 19, 503517, Berlin 2008.

Hecken, Thomas, Pop. Geschichte eines Konzeptes 1955-2009, Bielefeld 2009.

Huq, Rupa, Resistance or incorporation? Youth policy making and hip hop culture. In: Hodkinson, Paul; Deicke, Wolfgang, (eds.) Youth cultures: scenes, subcultures and tribes, Abingdon 2007, S. 79-92.

Jama, Zainab (2012). Spaceghost Purp, in: Juice 06/2012, S. 29-30.

Kage, Jan, American Rap. US - Hip Hop und Identität, Mainz 2002.

Käppner, Joachim, Das Kind, das nicht zurückkam, in: Süddeutsche Zeitung, 28./29.6.2008, München 2008, S. 8.

Lebert, Stephan; Willeke, Stefan, Ich mach dich fertig, ganz normal, in: Die Zeit 7, Hamburg 2008, S. 15-17.

Loh, Hannes und Güngör, Murat, Fear of a Kanak Planet. HipHop zwischen Weltkultur und Nazirap, Höfen 2002.

Luhmann, Niklas, Die Kunst der Gesellschaft, in: Werber, Nils (Hrsg.): Schriften zu Kunst und Literatur, Frankfurt a. M. 2008.

Mikos, Lothar, Interpolation and sampling. Kulturelles Gedächtnis und Intertextualität, in: Janis Androutsopoulus (Hrsg.): Globale Kultur - lokale Praktiken, Bielefeld 2003, S. 64-84. 
Moebius, Stephan und Schroer, Markus, Diven, Hacker, Spekulanten - Sozialfiguren der Gegenwart, Berlin 2010.

Robertson, Roland, Glokalisierung. Homogenität und Heterogenität in Raum und Zeit, in: Beck, Ulrich (Hrsg.), Perspektiven der Weltgesellschaft, Frankfurt a. M. 1998, S. 198220.

Rose, Tricia, Introduction, in: Rose, Tricia The wars of Hip Hop. What we talk about when we talk about hip hop - and why it matters. BasicCivitas, New York 2008, S. 33-61.

Seeliger, Martin, Kulturelle Repräsentation sozialer Ungleichheiten Eine vergleichende Betrachtung von Polit- und Gangsta-Rap, in: Dietrich, Marc; Seeliger, Martin (Hrsg.), Deutscher Gangstarap. Sozial- und kulturwissenschaftliche Perspektiven, Bielefeld 2012, 165-187.

Szillus, Stephan, ODD Future (Coverstory), in: Juice 06/2012, S. 20-27.

Toop, David, Rap Attack 3. African Rap to global Hip Hop, London 2000.

Weiß, Marco, Meine 247 Tage im türkischen Knast, Hamburg 2008.

\section{Internetquellen}

Dietrich, Marc und Seeliger, Martin (2012), Gangsta-Rap - mal wissenschaftlich. http://norient.com/de/academic/gangsta-rap/ (05.08.2012).

Esch, Christian (2012), Die Kirche hat das Nachsehen http://www.fr-online.de/meinung/analyse-zu-pussy-riot-die-kirche-hat-das-nachsehen, 1472602,16763688.html (05.08.2012).

Swedenburg, Ted: Islam in the mix. Lessons of the 5 Percent. http://comp.uark.edu/ tsweden/5per.html (05.08.2012).

Bartsch, Matthias (u. a.), Exempel des Bösen. In: Spiegel 2 / 2008. vgl. http://www.spiegel.de/spiegel/print/d-55294620.html (05.08.2012).

BZ-Artikel zu Deso Dogg BZ, http://www.bz-berlin.de/archiv/gesuchter-hass-rapper-untergetaucht-article1497573. html (05.08.2012).

Focus-Artikel zu Deso Dogg, http://www.focus.de/politik/deutschland/ex-rapper-deso-dogg-singt-loblied-auf-bin-lad en_aid_642789.html (05.08.2012).

\section{Musik, Albencover und Onlinevideos}

Big Daddy Kane im Playgirl http://www.blackdogue.net/Playgirl2/BigDaddyKane/BigDaddyKane.html (05.08.2012).

Big Daddy Kane "Smooth Operator" http://www.youtube.com/watch?v=8g5bjSUysQA (05.08.2012). 
Lyrics Paris "The devil made me do it"

http://www.lyricsmode.com/lyrics/g/golden_earring/the_devil_made_me_do_it.html (05.08.2012).

Video http://www.youtube.com/watch?v=hKRAFPHD8W4 (05.08.2012).

Deso Dogg Aufruf http://www.youtube.com/watch?v=i58F-mCTDDY\&feature $=$ related (05.08.2012).

ZDFNeo-Reportage Wild Germany zu Rap und Islam in Deutschland http://www.zdf.de/ZDFmediathek/hauptnavigation/startseite\#/beitrag/video/1285846/ Wild-Germany---Islamischer-Rap (05.08.2012).

Die universelle Flagge der 5 Percenter, unter: http://api.ning.com/files/-VCqNhXiur-XLob0ipFvknLsE6CpNbjkBOMjQDf6X9TcP6Qk QWkOkjobrfhURLGLn7X90sAwLcl7aF6o0N9J3HG9WRSYE6CI/UniversalFlag.gif (05.08.2012).

Das Albumcover von Rapper Lord Jamar (Brand Nubian), unter: http://www.bing.com/images/search?q = lord + jamar + the $+5+$ percent + album\&view $=$ detail\&id = D810C695E80B7FAC808AC15456310BEEA146396B\&FORM = IDFRIR (05.08.2012).

Das Albumcover zu „Sonic Jihad“ von Paris http://www.guerrillafunk.com/paris/sonic_jihad/ (05.08.2012). 